\title{
Briófitas na Estação Ecológica da Universidade Federal de Minas Gerais, Brasil
}

\author{
Gabriel Felipe Peñaloza-Bojacá, Laura Bubantz Fantecelle, \\ Cíntia Aparecida Teixeira Araújo \& Adaíses S. Maciel-Silva
}

\begin{abstract}
Universidade Federal de Minas Gerais, Instituto de Ciências Biológicas, Departamento de Botânica, Laboratório de Sistemática Vegetal, Av. Antônio Carlos, 6627, CEP 31270-901, Pampulha, Belo Horizonte, MG, Brasil. adaises.maciel@ufmg.br
\end{abstract}

Recebido em 08.IX.2015

Aceito em 26.IV. 2017

DOI: $10.21826 / 2446-8231201772105$

\begin{abstract}
RESUMO - A Estação Ecológica da Universidade Federal de Minas Gerais é uma importante unidade de conservação urbana com vegetação de Mata Semidecídua e Cerrado, sendo área de inúmeros estudos com a fauna e flora locais. No entanto, informações sobre briófitas são completamente ausentes, o que dificulta estudos mais aprofundados sobre estas plantas. O objetivo desse estudo foi registrar as espécies de briófitas presentes na Estação Ecológica da referida Universidade, apresentar informações sobre sua reprodução e dados complementares sobre o micro-habitat e distribuição geográfica das espécies. A partir de 60 amostras de briófitas coletadas em diferentes substratos foram encontradas nove espécies de hepáticas e 15 de musgos. O musgo Isopterygium byssobolax (Müll. Hal) Paris é novo registro para o estado, propágulos assexuados são descritos pela primeira vez em Donnellia lagenifera (Mitt.) W.R. Buck e a hepática Cylindrocolea planifolia (Steph.) R.M. Schust é novamente registrada para o estado após 160 anos.
\end{abstract}

Palavras-chave: diversidade, Cerrado, floresta semidecídua, reprodução

\begin{abstract}
Bryophytes in the Ecological Station of the Federal University of Minas Gerais, Brazil. The Ecological Station of the Federal University of Minas Gerais is an important urban conservation area with semi-deciduous forest and savanna vegetation where several studies on local fauna and flora have been carried out. However, there is no scientific information on bryophytes, which hinders detailed studies on these plants. The aim of this study was to record the bryophyte species present in the Ecological Station, and to add information on their reproduction, as well as supplementary data on microhabitats and geographic species distribution. Within 60 bryophyte samples collected in different substrates, nine liverwort species and fifteen moss species were found. The moss Isopterygium byssobolax (Mull.Hal.) Paris is a new record for the state of Minas Gerais; asexual propagules are described for the first time in the moss Donnellia lagenifera (Mitt.) W. R. Buck; and the liverwort Cylindrocolea planifolia (Steph.) R. M. Schust has again been recorded in the state after 160 years.
\end{abstract}

Keywords: diversity, Cerrado, forest semi-deciduous, reproduction

\section{INTRODUÇÃO}

O estado de Minas Gerais abarca dois importantes ecossistemas brasileiros, o Cerrado e a Mata Atlântica, que juntos representam $c a .30 \%$ da cobertura de vegetação nativa no estado (Machado et al. 2004, IEF 2015). A Universidade Federal de Minas Gerais dispõe de uma unidade de conservação urbana com vegetação típica de Mata Semidecídua e de Cerrado, denominada Estação Ecológica. O local possui área de 114 ha (UFMG 2015), contando com significativa diversidade de espécies de insetos, aracnídeos, mamíferos, anfíbios, répteis e aves, além de espécies vegetais, dentre elas nativas como a mutamba, cedro, ipê, cotieira e espécies exóticas como o eucalipto, a mangueira e o baobá (Souza-Silva et al. 2001, Kumagai 2002, Antonini \& Martins 2003, Álvares et al. 2004, Álvares et al. 2005, Julião et al. 2005, Tavares et al. 2010, Dias \& Kumagai 2011, UFMG 2015).

A importância deste espaço para o conhecimento e conservação da diversidade se reflete nas pesquisas desenvolvidas na Estação Ecológica da UFMG, com temas como análise e proteção ambiental (Dal Pont 2008), botânica (Barbosa-Campos 2011, Campos 2011, Oliveira et al. 2014), entomologia (Souza-Silva et al. 2001, Antonini \& Martins 2003, Loyola \& Martins 2008), fungos endofíticos (Oki et al. 2009) e metais pesados no solo (Belotti 2011). Além de estudos de cunho científico, a Estação Ecológica também compreende estudos de educação ambiental voltados à comunidade interna e externa à UFMG, por meio de oficinas dinâmicas, atividades lúdicas, trilhas ecológicas, entre outros, que são oferecidas aos visitantes (UFMG 2015). Dessa forma, a Estação Ecológica da UFMG é um importante espaço para desenvolvimento de atividades acadêmicas e educativas, permitindo a atuação de professores e alunos das diferentes áreas do saber.

Diante deste cenário, este trabalho apresenta-se como o primeiro estudo da comunidade de briófitas (hepáticas e musgos) ocorrente na Estação Ecológica da UFMG. O objetivo principal foi registrar a riqueza e composição de espécies, bem como caracterizá-las quanto ao microhabitat de ocorrência, formas de vida, distribuição geográfica e reprodução. Esses dados são importantes 
para o conhecimento da biodiversidade do estado de Minas Gerais, mas também proporcionarão maior difusão, em um contexto de educação ambiental, sobre a importância das briófitas como colonizadores primários, agentes contra a erosão do solo e mantenedoras de umidade local (Schofield 1985, Proctor 2000, Frahm 2003, Kürschner 2004).

\section{MATERIAL E MÉTODOS}

A Estação Ecológica (EEco) localiza-se no campus Pampulha da Universidade Federal de Minas Gerais na

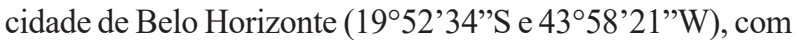
uma vegetação de Mata Atlântica e Cerrado, apresentando altitude média de $930 \mathrm{~m}$, pluviosidade anual de $1426 \mathrm{~mm}$, e temperatura anual de 14,6 a $26,8^{\circ} \mathrm{C}$ (Antonini \& Martins 2003, Hijmans et al. 2004).

Foram realizadas três excursões, com duração de $2 \mathrm{~h}$ cada, entre os meses de abril a setembro de 2015 ao longo de trilhas compreendidas em uma área de aproximadamente 14 ha. Essas trilhas compreenderam áreas com componentes de Floresta Semidecídua e Cerrado. Foram coletadas 60 amostras de briófitas, com área de pelo menos $4 \mathrm{~cm}^{2}$, a partir de diferentes substratos (casca viva, tronco morto caído, rocha e solo). As briófitas e seus substratos adjacentes foram coletados com auxílio de canivete e espátula, armazenados em sacos de papel devidamente etiquetados, e levados ao laboratório.

Em laboratório, todos os sacos foram mantidos abertos para secagem das amostras a temperatura ambiente. Algumas amostras de solo foram mantidas úmidas e cultivadas com luz artificial $\left(40 \mu \mathrm{mol} \cdot \mathrm{m}^{-2} \cdot \mathrm{s}^{-1}\right.$ e fotoperíodo de $\left.12 \mathrm{~h}\right)$. As amostras de briófitas foram umedecidas e analisadas sob estereomicroscópio e microscópio óptico. Quando esporófitos e estruturas de propagação vegetativa estiveram presentes, os mesmos foram registrados para cada espécime. Fotomicrografias foram obtidas para registros de táxons relevantes. Para identificação taxonômica, foram utilizados Fulford (1976), Buck (1990, 1998), Gradstein et al. (2001), Gradstein \& Costa (2003), Ireland \& Buck (2009) e outras referências contidas nelas. O sistema de classificação seguiu Crandall-Stotler et al. (2009) para hepáticas e Goffinet et al. (2009) para musgos. As exsicatas foram depositadas no herbário BHCB (17530 até 17558). A ocorrência por fitofisionomias brasileiras seguiu Costa et al. (2015), Costa (2015a; 2015b; 2015c, 2015d), Peralta (2015a, 2015b, 2015c, 2015d, 2015e, 2015f, 2015g), Câmara (2015), Henriques \& Costa (2015) e Bastos (2015). Informações sobre a distribuição geográfica das espécies no Brasil estão descritas pelas siglas dos respectivos Estados (AC - Acre; AL - Alagoas; AP - Amapá; AM - Amazonas; BA - Bahia; CE - Ceará; DF - Distrito Federal; GO - Goiás; MA Maranhão; MT - Mato Grosso; MS - Mato Grosso do Sul; MG - Minas Gerais; PA - Pará; PB - Paraíba; PR - Paraná; PE - Pernambuco; PI - Piauí; RJ - Rio de Janeiro; RN - Rio Grande do Norte; RS - Rio Grande do Sul; RO Rondônia; RR - Roraima; SC - Santa Catarina; SP - São Paulo; SE - Sergipe; TO - Tocantins) e seguiram a Flora do
Brasil. Em relação à distribuição mundial seguimos Allen (2002), Carmo, \& Peralta (2016), Costa (2017), Fulford (1976), Florschütz et al. (2011), Gradstein et al. (2001), Gradstein \& Costa (2003), Gradstein (2011), quando pertinente, outras referências são citadas nos comentários da espécies. Forma de vida e sistema sexual das espécies foram obtidos das referências citadas acima.

\section{RESULTADOS}

Foram encontradas nove espécies de hepáticas distribuídas em cinco famílias. As espécies mais abundantes foram Lejeunea laetevirens Nees \& Mont com oito espécimes e Frullania ericoides (Nees) Mont com seis espécimes. Para musgos, houve registro de 15 espécies distribuídas em 11 famílias. As espécies mais abundantes foram Chrysohypnum diminutivum (Hampe) W.R. Buck com 17 espécimes, Octoblepharum albidum Hedw com 16 e Sematophyllum subpinnatum (Brid.) E. Britton com 12. A maior ocorrência das espécies de hepáticas e de musgos citadas acima, pode ser justificada pelo conjunto de micro-habitats encontrados na EEco e pela ampla distribuição geográfica destas no Brasil, inclusive nos domínios fitogeográficos de Cerrado e Mata Semidecídua que estão presentes na EEco. Além disso, as espécies de hepática $L$. laetevirense, $F$. ericoides, assim como o musgo $C$. diminutivum são típicas de ambientes ensolarados (Gradstein 1992, Florschütz-de Waard \& Veling 1996, Gradstein et al. 2001, Santos et al. 2011) e os musgos O. albidum e $S$. subpinnatum são generalistas, ocorrendo tanto na sombra quanto no sol (Santos et al. 2011, Gradstein 1992).

O musgo Isopterygium byssobolax (Müll. Hal.) Paris (Fig. 1 a-d) é novo registro para o estado de Minas Gerais, ocorrendo também em São Paulo, Espírito Santo e Distrito Federal (ver comentários e distribuição detalhada abaixo na lista de espécies). Ele está presente nos domínios da Amazônia, Cerrado e Mata Atlântica segundo Peralta (2015e), portanto sua ocorrência na EEco era provável já que o local de estudo é uma área de transição entre os dois últimos ecossistemas.

Propágulos assexuados (Fig. 2 a-f) foram registrados pela primeira vez no musgo Donnellia lagenifera (Mitt.) W.R. (ver comentário detalhado na lista de espécies). Essas estruturas são filamentosas e frágeis, localizam-se nas axilas dos filídios e se originam do caulídio. Segundo Buck (1998), propágulos assexuados estão presentes apenas ocasionalmente em Sematophyllaceae; ocorrendo raramente, com exceção dos gêneros Aptychella e Wijkia. Para Donnellia, apenas a espécie Donnelia commutata (Müll.Hal.) W.R.Buck tinha sido registrada apresentando propágulos eventualmente. Por isso, a descrição destes propágulos é significativa para compreender os mecanismos de reprodução e dispersão de espécies de briófitas.

A hepática Cylindrocolea planifolia (Steph.) R.M. Schust. é registrada novamente para o estado de Minas Gerais após, aproximadamente, 160 anos (Fulford 1976). Costa \& Santos (2009) reconhecem C. planifolia como 

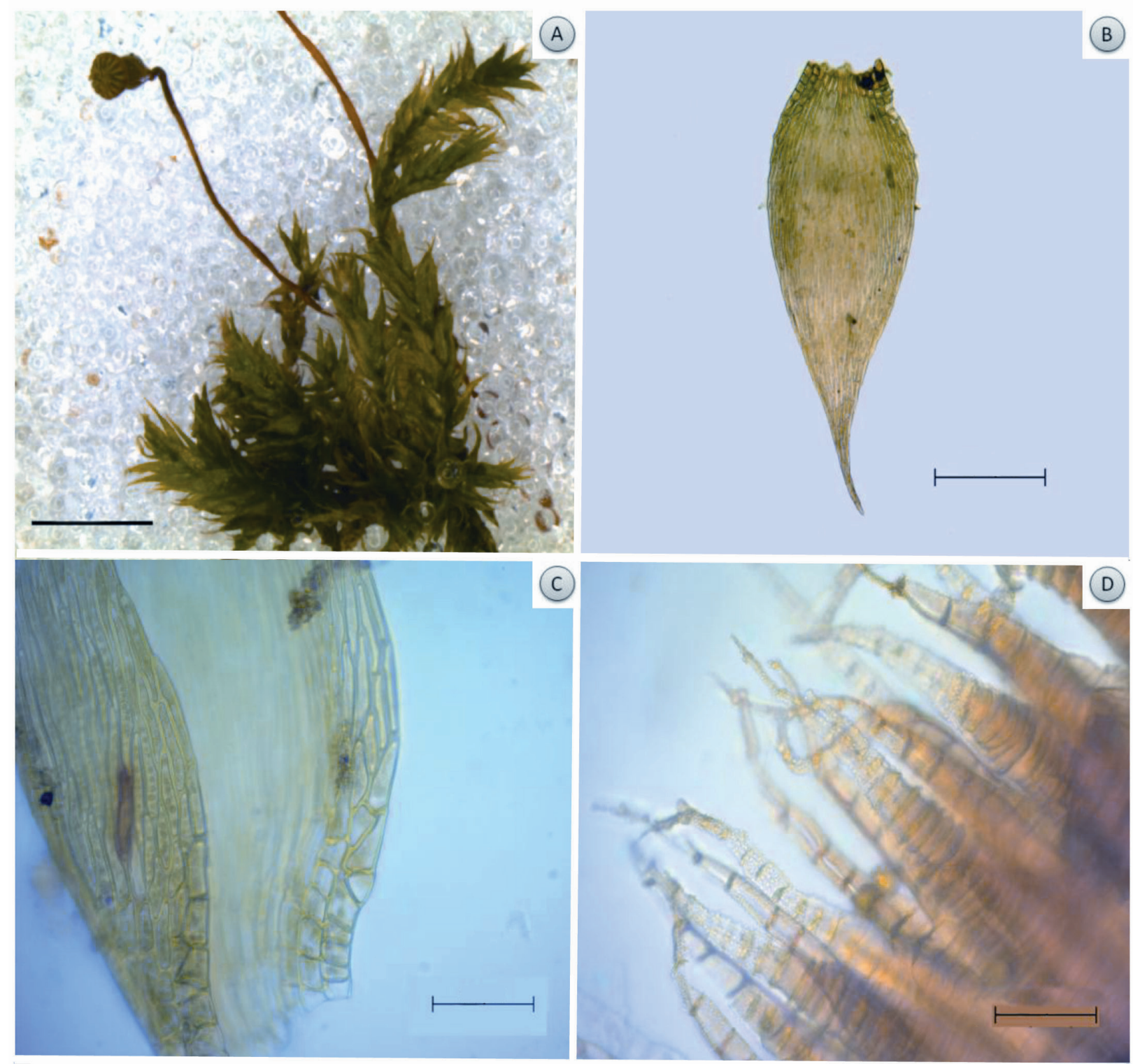

Figs 1 A-D. Isopterygium byssobolax (Müll. Hal.) Paris. A. Hábito de crescimento; B. Filídio; C. Região basal do filídio; D. Dentes do peristômio. Barras: $\mathbf{A}=1 \mathrm{~mm} ; \mathbf{B}=200 \mu \mathrm{m} ; \mathbf{C}-\mathbf{D}=50 \mu \mathrm{m}$.

espécie vulnerável para o estado do Rio de Janeiro; entretanto devido a seu hábito, que é comum próximo a curso de água em ambiente sombreado, e também ao pequeno tamanho variando entre 0,5 e $1 \mathrm{~mm}$ de comprimento (Fig. 3 a-c; Gradstein \& Costa 2003), a espécie pode ser sub amostrada. De qualquer forma, este é um registro relevante para o estado de Minas Gerais e demonstra parcela importante da biodiversidade que a EEco pode abrigar.

Abaixo segue a listagem das espécies de hepáticas (Marchantiophyta) e de musgos (Bryophyta) encontradas na Estação Ecológica da UFMG. Registros novos para o estado são destacados em negrito (MG). Informações pertinentes sobre substrato, associação com outras espécies de briófitas e distribuição das espécies são apresentadas. Comentários detalhados sobre as espécies são feitos para os registros mais relevantes.

\section{Marchantiophyta}

Aneuraceae

Riccardia amazonica (Spruce) Schiffn. ex Gradst. \& Hekking. J. Hattori Bot. Lab. 45: 129. 1979.

Material examinado: BRASIL, MINAS GERAIS, Belo Horizonte, Estação Ecológica da UFMG, V.2014, A.S. Maciel-Silva 285 (BHCB 175336).

Distribuição: Espécie afro-americana. Registrada para os estados de AM, AP, PA, AL, BA, PE, GO, ES, MG, RJ e SP.

Domínio fitogeográfico: Amazônia, Cerrado e Mata Atlântica.

Comentários: Espécie talosa em tapete, dioica, sem 

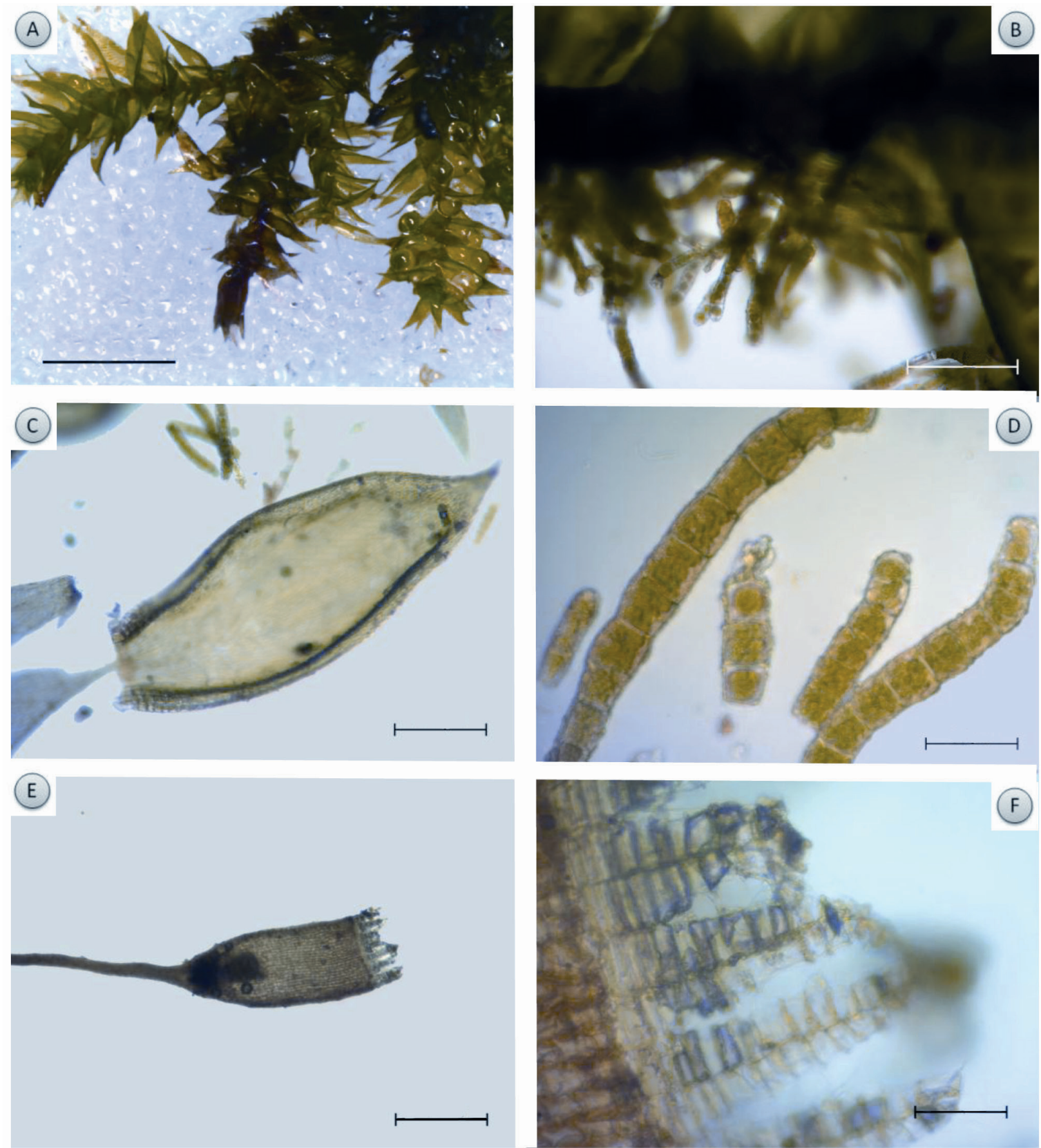

Figs. 2 A-F. Donnellia lagenifera (Mitt.) W.R. Buck. A. Hábito de crescimento mostrando filídios falcados; B. Propágulos assexuados nas axilas dos filídios; C. Filídio profundamente côncavo; D. Propágulos destacados e cápsula; E, F. Dentes do peristômio. Barras: A=1 mm; B-C=200 $\mu \mathrm{m}$; $\mathbf{D}, \mathbf{F}=50 \mu \mathrm{m} ; \mathbf{E}=500 \mu \mathrm{m}$

estruturas reprodutivas sexuadas e assexuadas no material examinado. Encontrada em solo de argila. Apresentou associações com Cylindrocolea planifolia e Fossombronia porphyrorhiza (Nees) Prosk.

\section{Cephaloziellaceae}

Cylindrocolea planifolia (Steph.) R.M. Schust. Nova Hedwigia 22: 164. 1971.

Material examinado: BRASIL, MINAS GERAIS, Belo
Horizonte, Estação Ecológica da UFMG, VIII.2014, A.S. Maciel-Silva 306 (BHCB 175357).

Distribuição: Espécie neotropical. Registrada para os estados de AC, RO, BA, MG, RJ, SP e SC.

Domínio fitogeográfico: Amazônia e Mata Atlântica.

Comentários: Espécie folhosa formando trama, monoica (autoica), com esporófito no material coletado. Apresentou 


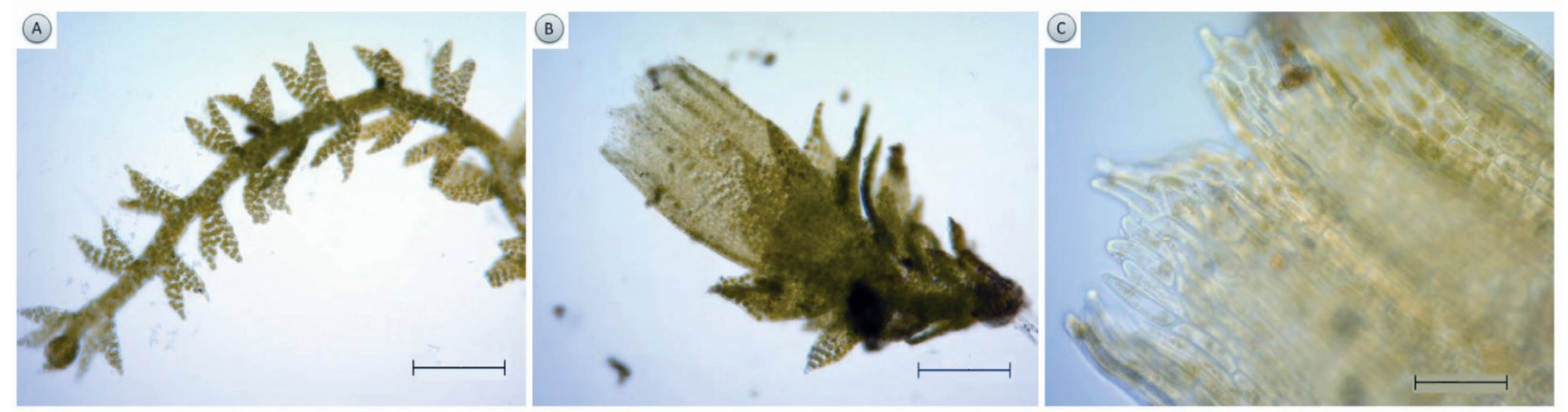

Figs 3. A-C. Cylindrocolea planifolia (Steph.) R.M. Schust. A. Hábito de crescimento; B. ginoécio; C. detalhe da boca do perianto. Barras: A-B= $200 \mu \mathrm{m} ; \mathbf{C}=50 \mu \mathrm{m}$.

associações com Riccardia amazonica e Fossombronia porphyrorhiza. Encontrada em solo. Caracteriza-se por possuir plantas de 0,5 a 1,0 mm de largura, ramos sexuais femininos (ginoécios) sobre ramos curtos e boca do perianto formada por células alongadas (Costa et al. 2008; Fig. 3 a-c).

\section{Fossombroniaceae}

Fossombronia porphyrorhiza (Nees) Prosk. Bryologist 58: 197. 1955.

Material examinado: BRASIL, MINAS GERAIS, Belo Horizonte, Estação Ecológica da UFMG, V.2014, A.S. Maciel-Silva 285 (BHCB 175336).

Distribuição: Espécie tropical america. Registrada para os estados de TO, BA, CE, MA, PE, PI, DF, GO, MT, MS, ES, MG, RJ e SP.

Domínio fitogeográfico: Caatinga, Cerrado, Mata Atlântica e Pantanal.

Comentários: Espécie folhosa em tapete, monoica (autoica), apresentando esporófito no material coletado. Encontrada em solo. Exibiu associação com as espécies Cylindrocolea planifolia e Riccardia amazonica.

\section{Frullaniaceae}

Frullania ericoides (Nees) Mont. Ann. Sci. Nat., Bot., sér. 2 12: 51.1839.

Material examinado: BRASIL, MINAS GERAIS, Belo Horizonte, Estação Ecológica da UFMG, V-VIII.2014 A.S. Maciel-Silva 273, 294 e 295 (BHCB 175324, 175345 e 175346).

Distribuição: Espécie pantropical. Registrada para os estados de AC, AM, PA, AL, BA, CE, MA, PB, PE, SE, DF, GO, MS, MT, ES, MG, RJ, SP, PR, RS e SC.

Domínio fitogeográfico: Amazônia, Caatinga, Cerrado, Mata Atlântica, Pampa e Pantanal.

Comentários: Espécie folhosa em trama, sem estruturas reprodutivas sexuadas e assexuadas no material examinado. Encontrada em casca de árvore. Exibiu associação com as espécies Sematophyllum subpinnatum, Erythrodontium squarrosum (Hampe) Paris, Brachiolejeunea phyllorhiza (Nees) Kruijt \& Gradst., Frullanoides liebmanniana (Lindenb. \& Gottsche), Chryso-hypnum diminutivum e Syrrhopodon ligulatus Mont..

\section{Lejeuneaceae}

Brachiolejeunea phyllorhiza (Nees) Kruijt \& Gradst. Nova Hedwigia 43: 299. 1986.

Material examinado: BRASIL, MINAS GERAIS, Belo Horizonte, Estação Ecológica da UFMG, V.2014, A.S. Maciel-Silva 265, 274 e 279 (BHCB 175316, 175325 e 175330).

Distribuição: Espécie afro-americana. Registrada para os estados de AM, BA, PE, ES, MG, RJ e SP.

Domínio fitogeográfico: Caatinga, Cerrado, Mata Atlântica, Pantanal.

Comentários: Espécie folhosa em tapete, monoica (autoica). O material coletado apresentou perianto e esporófito. Encontrada em casca de árvore. Em associação com Sematophyllum subpinnatum, Erythrodontium squarrosum e Frullania ericoides.

Cheilolejeunea discoidea (Lehm. \& Lindenb.) Kachr. \& R.M. Schust. J. Linn. Soc., Bot. 56: 509. 1961.

Material examinado: BRASIL, MINAS GERAIS, Belo Horizonte, Estação Ecológica da UFMG, V-VIII.2014, A.S. Maciel-Silva 269, 270, 297 e 301(BHCB 175320, 175321,175348 e 175352).

Distribuição: Espécie neotropical. Registrada para os estados de BA, SE, DF, GO, MS, MT, ES, MG e SP.

Domínio fitogeográfico: Cerrado, Mata Atlântica e Pantanal.

Comentários: Espécie folhosa em tapete, monoica 
(autoica), com presença de perianto e esporófito no material coletado. Encontrada em casca de árvore. Possui associação com as espécies Ochrobryum gardneri (Müll.Hal.) Lindb., Octoblepharum albidum e Lejeunea laetevirens.

Frullanoides liebmanniana (Lindenb. \& Gottsche) Slageren. Meded. Bot. Mus. Herb. Rijks Univ. Utrecht 544: 102. 1985.

Material examinado: BRASIL, MINAS GERAIS, Belo Horizonte, Estação Ecológica da UFMG, V.2014, A.S. Maciel-Silva 303 (BHCB175354).

Distribuição: Espécie neotropical. Registrada para os estados de AC, PE, GO, MT, MG, SP e PR.

Domínio fitogeográfico: Amazônia, Cerrado e Mata Atlântica.

Comentários: Espécie folhosa em tapete, dioica, com perianto e esporófito no material coletado. Espécie encontrada em casca de árvore. Exibiu associação com Sematophyllum subpinnatum, Frullania ericoides e Stereophyllum radiculosum (Hook.) Mitt.

Lejeunea caulicalyx (Stephani) M. E. Reiner \& Goda. J. Hattori Bot. Lab. 89: 13. 2000.

Material examinado: BRASIL, MINAS GERAIS, Belo Horizonte, Estação Ecológica da UFMG, V.2014, A.S. Maciel-Silva 252 (BHCB 175303).

Distribuição: Espécie neotropical. Registrada para os estados de AC, PA, RR, AL, BA, PE, MS, MT, ES, MG, RJ, SP, PR; além de AM, PA, CE, DF, GO, RS e SC como Lejeunea glaucescens Gottsche.

Domínio fitogeográfico: Amazônia, Cerrado, Mata Atlântica e Pantanal.

Comentários: Espécie folhosa em tapete, monoica (autoica), com perianto no material coletado. Em associação com Lejeunea laetevirens. Caracteriza-se pelos lóbulos dos filídios sempre pequenos, reduzidos a vestigiais, algumas vezes inflados e anfigastros pequenos. Encontrada em casca de árvores. Apesar de aqui ser tratada como registro novo para o estado, os registros anteriores de L. glaucescens podem ser, na verdade, referentes a Lejeunea caulicalyx (ver Reiner-Drehwald \& Goda 2000, Bastos \& Yano 2009, Yano et al. 2011).

Lejeunea laetevirens Nees \& Mont. Hist. Phys. Cuba, Bot., Pl. Cell. 469. 1842.

Material examinado: BRASIL, MINAS GERAIS, Belo Horizonte, Estação Ecológica da UFMG, V.2014, A.S. Maciel-Silva 257 e 262 (BHCB 175308 e 175313).
Distribuição: Espécie neotropical. Registrada para os estados de AC, AM, AP, PA, RR, AL, BA, CE, MA, PB, $\mathrm{PE}, \mathrm{RN}, \mathrm{SE}, \mathrm{DF}, \mathrm{GO}, \mathrm{MS}, \mathrm{MT}, \mathrm{ES}, \mathrm{MG}$ por Tonini et al. (2005) e Yano (2010), RJ, SP, PR, RS e SC.

Domínio fitogeográfico: Amazônia, Caatinga, Cerrado, Mata Atlântica e Pantanal.

Comentários: Espécie folhosa em tapete, dioica, e com perianto no material examinado. Encontrada em casca de árvore. Associação com Leptostomopsis systylium (Müll. Hal.) J.R.Spence \& H.P.Ramsay, Octoblepharum albidum, Isopterygium tenerum (Sw.) Mitt. J. Linn, Cheilolejeunea discoidea e Donnellia lagenifera. Caracteriza-se pelos anfigastros distantes, e por filídios dispostos obliquamente no caulídio, com ápice obtuso e cutícula finamente papilhosa (Reiner-Drehwald 2000, Reiner-Drehwald 2003, Bastos \& Yano 2009).

\section{Bryophyta}

Bryaceae

Leptostomopsis systylia (Müll. Hal.) J.R. Spence \& H.P. Ramsay. Phytologia 87(2): 70. 2005.

Material examinado: BRASIL, MINAS GERAIS, Belo Horizonte, Estação Ecológica da UFMG, V.2014, A. S. Maciel-Silva 257 (BHCB 175308).

Distribuição: Espécie pantropical. Registrada para os estados de AL por Yano et al. (2010), BA, DF, ES, RJ e MG por Tonini et al. (2005).

Domínio fitogeográfico: Cerrado e Mata Atlântica.

Comentários: Espécie acrocárpica em tufo, dioica, sem esporófito ou estruturas reprodutivas assexuadas no material examinado. Encontrada em casca de árvore. Apresentou associação com Lejeunea laetevirens.

Calymperaceae

Octoblepharum albidum Hedw. Sp. Musc. Frond. 50. 1801.

Material examinado: BRASIL, MINAS GERAIS, Belo Horizonte, Estação Ecológica da UFMG, V-VIII.2014, A.S. Maciel-Silva 255, 259, 271, 275, 281, 282, 286, 296, 300, 305 (BHCB 175306, 175310, 175322 175326, 175332, $175333,175337,175347,175351$ e 175356$)$.

Distribuição: Espécie pantropical. Registrada para os estados de AC, AM, AP, PA, RO, RR, TO, AL, BA, CE, MA, PB, PE, PI, RN, SE, DF, GO, MS, MT, ES, RJ, MG, $\mathrm{SP}, \mathrm{PR}, \mathrm{RS}$ e SC.

Domínio fitogeográfico: Amazônia, Caatinga, Cerrado, Mata Atlântica, Pampa e Pantanal.

Comentários: Espécie acrocárpica em tufos, monoica 
(autoica), exibiu esporófito e estruturas de propagação vegetativa no material examinado. Encontrada em casca de árvore e tronco em decomposição. Apresentou associação com Donnellia lagenifera, lejeunea laetevirens, Sematophyllum subpinnatum, Chryso-hypnun diminutivum, Cheilolejeunea discoidea, Isopterygiun tenerum e Syrrhopodon ligulatus.

Syrrhopodon ligulatus Mont. Syll. Gen. Sp. Crypt. 47. 1856.

Material examinado: BRASIL, MINAS GERAIS, Belo Horizonte, Estação Ecológica da UFMG, VIII.2014, A.S. Maciel-Silva 299 e 304 (BHCB 175350 e 175355).

Distribuição: Espécie neotropical. Registradas para os estados de AC, AM, AP, PA, RO, RR, BA, PE, DF, GO, MS, MT, MG, RJ e SP.

Domínio fitogeográfico: Amazônia, Cerrado e Mata Atlântica.

Comentários: Espécie acrocárpica em tufos, dioica, exibiu esporófito no material coletado. Encontrada em casca de árvore. Apresentou associação com a espécie Syrrhopodon ligulatus, Isopterygiun tenerum, Octoblepharum albidum, Frullania ericoides, Sematophyllum subpinnatum e Chrysohypnun diminutivum.

Entodontaceae

Erythrodontium squarrosum (Hampe) Paris. Index Bryol. (ed. 2) 159. 1904.

Material examinado: BRASIL, MINAS GERAIS, Belo Horizonte, Estação Ecológica da UFMG, V.2014, A.S. Maciel-Silva 260, 263, 290 e 293 (BHCB 175311, 175314, 175341 e 175344).

Distribuição: Pantropical. Registrada para os estados de PA, DF, GO, MS, MT, ES, MG, RJ, SP, PR, RS e SC.

Domínio fitogeográfico: Amazônia, Cerrado, Mata Atlântica e Pantanal.

Comentários: Espécie pleurocárpica em tapete, presente em casca de árvore, monoica (autoica), e exibiu esporófito no material examinado. Encontrada em casca de árvore. Apresentou associação com Frullania ericoides, Sematophyllum subpinnatum, Chryso-hypnun diminutivum e Syrrhopodon ligulatus.

\section{Erpodiaceae}

Erpodium glaziovii Hampe. Vidensk. Meddel. Dansk Naturhist. Foren. Kjøbenhavn 4: 54. 1872.

Material examinado: BRASIL, MINAS GERAIS, Belo Horizonte, Estação Ecológica da UFMG, VIII.2014, A.S. Maciel-Silva 302 (BHCB 175353).
Distribuição: Espécie pantropical. Registrada para os estados de AM, BA, MS, MT, ES, RJ, MG, SP, PR, RS e SC.

Domínio fitogeográfico: Amazônia, Cerrado, Mata Atlântica, Pampa e Pantanal.

Comentários: Espécie pleurocárpica em trama, monoica (autoica) e exibiu esporófito no material examinado. Encontrada em casca de árvore. Apresentou associação com Sematophyllum subpinnatum, Frullania ericoides e Brachiolejeunea phyllorhiza.

Helicophyllaceae

Helicophyllum torquatum (Hook.) Brid. Bryol. Univ. 2: 771.1827.

Material examinado: BRASIL, MINAS GERAIS, Belo Horizonte, Estação Ecológica da UFMG, V.2014, A.S. Maciel-Silva 292 (BHCB 175343).

Distribuição: Espécie neotropical. Registrada para os estados de AM, PA, TO, AL, BA, CE, PB, PE, PI, GO, MS, MT, ES, MG, RJ, SP, PR e SC.

Domínio fitogeográfico: Amazônia, Caatinga, Cerrado, Mata Atlântica, Pampa e Pantanal.

Comentários: Espécie pleurocárpica em trama, monoica (autoica), sem esporófito e estruturas reprodutivas assexuadas no material examinado. Encontrada em casca de árvore. Apresentou associação com Sematophyllum subpinnatum e Brachiolejeunea phyllorhiza.

\section{Hypnaceae}

Chryso-hypnum diminutivum (Hampe) (Hampe) W.R. Buck. Brittonia 36(2): 182. 1984.

Material examinado: BRASIL, MINAS GERAIS, Belo Horizonte, Estação Ecológica da UFMG, V-VIII.2014. A.S. Maciel-Silva 249, 253, 254, 258, 261, 266, 267, 268, 278, 280, 283, 284, 287, 288 e 307 (BHCB 175300, 175304, 175305, 175309, 175312, 175317, 175318, 175319, 175329, 175331, 175334, 175335, 175338, 175339 e 175358).

Distribuição: Espécie neotropical. Registrada para os estados de AC, AM, AP, PA, RO, TO, BA, PE, DF, GO, MS, MT, ES, MG, RJ, SP, PR, RS e SC.

Domínio fitogeográfico: Amazônia, Cerrado, Mata Atlântica, Pampa e Pantanal.

Comentários: Espécie pleurocárpica em trama, monoica (autoica) e exibiu esporófito no material coletado. Encontrada em casca de árvore, tronco em decomposição e em solo de argila. Apresentou associação com Octoblepharum albidum, Sematophyllum subpinnatum, Syrrhopodon ligulatus e Frullania ericoides. 
Leucobryaceae

Ochrobryum gardneri (Müll. Hal.) Mitt. J. Linn. Soc., Bot. 12: 108. 1869.

Material examinado: BRASIL, MINAS GERAIS, Belo Horizonte, Estação Ecológica da UFMG, V.2014, A.S. Maciel-Silva 270 (BHCB 175321).

Distribuição: Espécie Neotropical e africa. Registrada para os estados de AM, PA, RO, RR, TO, AL, BA, CE, PE, DF, GO, MS, MT, ES, MG, RJ, SP e PR.

Domínio fitogeográfico: Amazônia, Cerrado, Mata Atlântica e Pantanal.

Comentários: Espécie acrocárpica em tufos, dioica, e com estruturas de propagação vegetativa (gemas nos filídios) no material examinado. Encontradas em casca de árvore. Expõe associação com Cheilolejeunea discoidea e Donnellia lagenifera.

\section{Pottiaceae}

Hyophila involuta (Hook.) A. Jaeger. Ber. Thätigk. St. Gallischen Naturwiss. Ges. 1871-72: 354 (Gen. Sp. Musc. 1: 202). 1873.

Material examinado: BRASIL, MINAS GERAIS, Belo Horizonte, Estação Ecológica da UFMG, V.2014, A.S. Maciel-Silva 256, 264, 272, e 289 (BHCB 175307175315 175323 e 175340).

Distribuição: Espécie Cosmopolita. Registrada para os estados de AM, PA, RO, RR, AL, BA, CE, MA, PB, PE, PI, DF, GO, MS, MT, ES, MG, RJ, SP, PR e RS.

Domínio fitogeográfico: Amazônia, Caatinga, Cerrado, Mata Atlântica, Pampa e Pantanal.

Comentários: Espécie acrocárpica em tufos, dioica, e com esporófito e estruturas de propagação vegetativa no material examinado. Encontra em rocha em concreto e solo de argila.

\section{Pylaisiadelphaceae}

Isopterygium byssobolax (Müll.Hal.) Paris. Index Bryol. Suppl. 248. 1900.

Material examinado: MINAS GERAIS, Belo Horizonte, Estação Ecológica da UFMG, V.2014, A.S. Maciel-Silva 291 (BHCB 175342).

Distribuição: Espécie neotropical. Registrada para os estados de RO, DF, MT, ES, SP, MG, PR, RS e SC.

Domínio fitogeográfico: Amazônia, Cerrado e Mata Atlântica.
Comentários: Encontrada em casca de árvore, pleurocárpica em tapete, monoica (autoica), apresentou esporófito no material examinado. Caracteriza-se por plantas pequenas até $1,3 \mathrm{~cm}$ de comprimento, filídios acuminados, células alares curtas retangulares a quadradas ou transversalmente alongadas em duas a quatro fileiras com quatro a dez células nas margens (Ireland \& Buck 2009; Fig. 1 a-d).

Isopterygium tenerum (Sw.) Mitt. J. Linn. Soc., Bot. 12: 499. 1869.

Material examinado: BRASIL, MINAS GERAIS, Belo Horizonte, Estação Ecológica da UFMG, V.2014 e VIII.2014, A.S. Maciel-Silva 277 e 298 (BHCB 175328 e 175349)

Distribuição: Espécie Cosmopolita. Registrada para os estados de AC, AM, PA, RO, RR, TO, BA, CE, MA, PB, PE, PI, DF, GO, MS, MT, ES, MG, RJ, SP, PR, RS e SC.

Domínio geográfico: Amazônia, Caatinga, Cerrado, Mata Atlântica, Pampa e Pantanal.

Comentários: Espécie pleurocárpica em tapete, monoica (autoica), exibiu esporófito no material examinado. Encontrada em casca de árvore. Apresentou associação com Octoblepharum albidum, Lejeunea laetevirens e Syrrhopodon ligulatus.

\section{Sematophyllaceae}

Donnellia lagenifera (Mitt.) W.R. Buck. Bryologist 91: 134. 1988.

Material examinado: BRASIL, MINAS GERAIS, Belo Horizonte, Estação Ecológica da UFMG, V.2014, A.S. Maciel-Silva 251 (BHCB175302).

Distribuição: Espécie endêmica do Brasil. Registrada para os estados de PA, MG, RJ, SP, RS e SC.

Domínio fitogeográfico: Amazônia e Mata Atlântica.

Comentários: Encontrada em casca de árvore. Espécie pleurocárpica em tapete, monoica (autoica), exibiu esporófito e estruturas de propagação vegetativa no material examinado. Apresentou associação com Cheilolejeunea discoidea, Ochrobryum gardneri, Octoblepharum albidum e Lejeunea laetevirens. Caracteriza-se pelos filídios espalhados a esquarrosos quando úmidos, acuminados, e fortemente côncavos especialmente na base; as células alares são menores que o dobro da largura das supra-alares e coloridas (Buck, 1994). Pela primeira vez são descritas estruturas de propagação vegetativa para a espécie. Os propágulos são filamentosos, encontrados nas axilas dos filídios, formados por de três a 12 células clorofiladas e com parede rugosa (Fig. 2 b,d). 
Sematophyllum galipense (Müll. Hal.) Mitt. J. Linn. Soc., Bot. 12: 480. 1869.

Material examinado: BRASIL, MINAS GERAIS, Belo Horizonte, Estação Ecológica da UFMG, V.2014, A.S. Maciel-Silva 276 (BHCB 175327).

Distribuição: Espécie afro-americana. Registrada para os estados de PA, TO, BA, CE, MA, DF, GO, MT, ES, MG, RJ, SP, RS e SC.

Domínio fitogeográfico: Amazônia, Cerrado, e Mata Atlântica.

Comentários: Encontrada em solo. Espécie pleurocárpica em tapete, monoica (autoica), e não apresentou esporófito ou estruturas de reprodução assexuada no material coletado.

Sematophyllum subpinnatum (Brid.) E. Britton. Bryologist 21(2): 28. 1918.

Material examinado: BRASIL, MINAS GERAIS, Belo Horizonte, Estação Ecológica da UFMG, V.2014, A.S. Maciel-Silva 250 (BHCB 175301).

Distribuição: Espécie de distribuição ampla. Registrada para os estados de AC, AM, PA, RO, RR, TO, AL, BA, CE, MA, PE, DF, GO, MS, MT, ES, MG, RJ, SP, PR, RS e SC.

Domínio fitogeográfico: Amazônia, Caatinga, Cerrado, Mata Atlântica, Pampa e Pantanal.

Comentários: Espécie pleurocárpica em tapete, monoica (autoica), e no material examinado apresentou esporófito. Encontrada em tronco de árvore em decomposição. Apresentou associação com Chryso-hypnum diminutivum, Syrrhopodon ligulatus, Frullania ericoides, Erythrodontium squarrosum, Fossombronia porphyrorhiza, Helicophyllum torquatum, Frullanoides liebmanniana, Stereophyllum radiculosum e Octoblepharum albidum.

\section{Stereophyllaceae}

Stereophyllum radiculosum (Hook.) Mitt. J. Linn. Soc., Bot. 12: 542. 1869.

Material examinado: BRASIL, MINAS GERAIS, Belo Horizonte, Estação Ecológica da UFMG, VIII.2014, A.S. Maciel-Silva 303 (BHCB 175354).

Distribuição: Espécie de distribuição pamtropical. Registrada para os estados de PA, BA, GO, MS, MT, ES, MG, RJ, SP, PR e SC.

Domínio fitogeográfico: Amazônia, Cerrado, Mata Atlântica e Pantanal.

Comentários: Espécie pleurocárpica em trama, monoica (autoica) e com esporófito no material examinado. Encontrada em casca de árvore. Apresentou associação com as espécies Sematophyllum subpinnatum, Frullania ericoides e Frullanoides liebmanniana.

\section{DISCUSSÃO}

As briófitas coletadas na Estação Ecológica da UFMG ocorreram em três substratos: casca de árvore viva (71\% dos espécimes), seguido de solo/rocha e tronco em decomposição (Fig. 4). A predominância de briófitas em tronco vivo também é relatada por diferentes autores como Aquino et al. (2015) e Sousa \& Câmara (2015) estudando matas de galeria no Cerrado brasileiro; por Holz et al. (2002) e Holz \& Gradstein (2005) em florestas montanas na Costa Rica; por Alvarenga \& Lisboa (2009), Ilkiu-Borges et al. (2009), Moura et al. (2013) e Brito \& Ilkiu-Borges (2014) em ecossistemas amazônicos; e Costa (1999) em florestas de terras baixas no sudeste do Brasil. Troncos vivos, assim como troncos em decomposição, são substratos favoráveis à colonização de briófitas pois retêm elevada umidade e geralmente estão disponíveis no ambiente, sendo colonizados por diferentes espécies de briófitas, desde a base até o dossel superior (Holz et al. 2002, Holz $\&$ Gradstein 2005).

$\mathrm{Na}$ EEco troncos em decomposição não são tão abundantes quanto troncos vivos, o que explica a menor representatividade da comunidade de briófitas neste substrato comparada à comunidade terrestre. Entre os registros relevantes, fazem parte da comunidade epifítica e epixílica os musgos Donnellia lagenifera, que segundo Buck (1994) cresce normalmente nos galhos das árvores e também com menor frequência sobre troncos; e o musgo Isopterygium byssobolax, que de acordo com Ireland \& Buck (2009) cresce em madeira em decomposição e árvores vivas.

O segundo substrato em ocorrência de espécimes (21\%) foi o solo/rocha, cujas amostras de briófitas foram coletadas principalmente nas bordas da mata ao longo de

Tipo de substrato

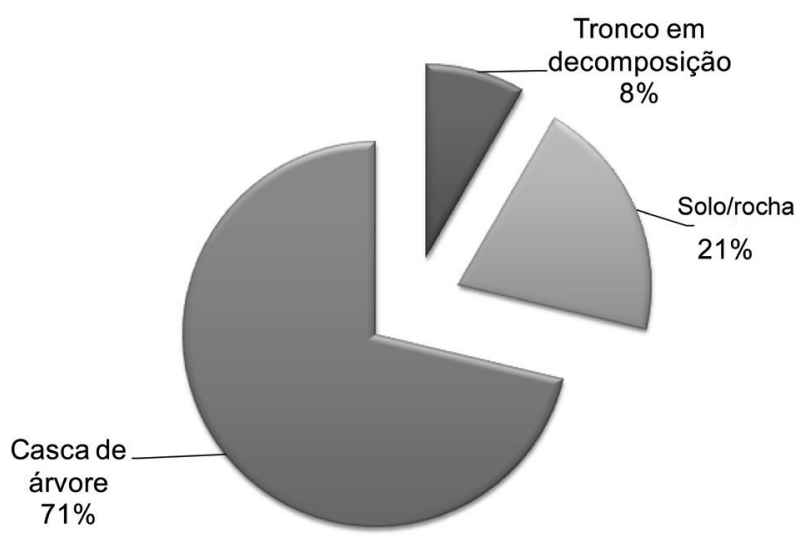

Fig. 4. Porcentagem de briófitas coletadas em diferentes substratos da Estação Ecológica da UFMG (Belo Horizonte, Minas Gerais). 
trilhas e em clareiras. Neste estudo, visto que apenas o musgo Hyophila involuta (Hook.) A.Jaeger ocorreu sobre rocha associada a concreto, mas também se estendendo ao solo, optou-se por representar esses dois substratos juntos. Houve pouca expressividade da comunidade de briófitas terrestres no interior da mata, o que se deve provavelmente à deposição e acúmulo de serrapilheira, levando a uma composição taxonomicamente mais restrita neste substrato (Gradstein 1997; Germano 2003; Alvarenga \& Lisboa, 2009). A hepática Cylindrocolea planifolia foi encontrada em casca de árvore e solo, concordando com Fulford (1976) que a registrou anteriormente nestes mesmos substratos.

A maioria das espécies de hepáticas (c.78\%) e musgos $(60 \%)$ foi encontrada com esporófitos, enquanto que a propagação vegetativa foi pouco expressiva $(<15 \%)$ nos dois grupos (Fig. 5). Quanto ao sistema sexual das espécies, as hepáticas apresentaram $67 \%$ das espécies monoicas e $33 \%$ dioicas (6 e 3 espécies, respectivamente). Do mesmo modo, os musgos foram representados por $74 \%$ (11 spp.) monoicas e $26 \%$ (4 spp.) dioicas.

Sete espécies de hepáticas apresentaram esporófitos (6 monoicas vs. 1 dioica). A hepática com maior abundância na área de estudo, Lejeunea laetevirens (dioica), apesar de possuir perianto, não apresentou esporófitos nas amostras analisadas. Segundo Reiner-Drehwald (2000) essa espécie é normalmente encontrada estéril, mas propaga-se vegetativamente por meio da fragmentação dos ramos.

Entre os musgos, nove espécies apresentaram esporófitos, sendo três delas as mais abundantes na EEco e também monoicas, Chryso-hypnum diminutivum, Octoblepharum albidum e Sematophyllum subpinnatum. Os musgos Hyophila involuta (dioica) e Donnellia lagenifera (monoica), apresentaram tanto esporófitos quanto estruturas de reprodução assexuada; e o musgo Ochrobryum gardneri (dioico) foi encontrado apenas com gemas, concordando com o descrito por Allen (1992) a respeito da ocorrência no gênero de propágulos sobre a superfície dos filídios.

O sistema sexual mais significativo na Estação Ecológica foi o monoico, com mais de $60 \%$ das espécies de hepáticas e dos musgos analisados. Espécies de briófitas monoicas são geralmente encontradas com esporófitos (Longton 1992, Oliveira \& Pôrto 1998, Maciel-Silva et al. 2012). Esse padrão deve-se possivelmente à proximidade entre os gametângios nos ramos de uma mesma planta, o que permite maiores chances de fecundação (Glime 2013). Além disso, a maior frequência de espécies monoicas na área de estudo pode ter relação com a produção constante de esporófitos e seus respectivos esporos, o que favorece a dispersão e estabelecimento de novos indivíduos em escala local (Alvarenga et al. 2009).

As espécies encontradas na EEco mostram, em geral, uma distribuição ampla com registros para todos os ecossistemas brasileiros. Por outro lado, a hepática Cylindrocolea planifolia apresenta distribuição disjunta na Amazônia e Mata Atlântica, e a hepática Brachiolejeunea phyllorhiza só é registrada para o domínio da Mata Attântica (Costa 2015b, Costa et al. 2015). Esses dois registros são, de certa forma, coerentes com os ecossistemas presentes no local de estudo e ampliam a distribuição das espécies para uma zona de transição entre Mata Atlântica e Cerrado.

As espécies registradas foram caracterizadas em cinco padrões de distribuição mundial (Fig. 6), o Neotropical foi o predominante com dez espécies, seguido do Pantropical com seis espécies, o Afro-americano com cinco espécies, Amplo com duas espécies, e uma única espécie endêmica do Brasil. Entre as espécies mais abundantes, Chryso-hypnum diminutivum e Lejeunea laetevirens têm distribuição neotropical, enquanto Octoblepharum albidum e Frullania ericoides são pantropicais. O musgo Donnellia lagenifera foi a única espécie endêmica para o Brasil.

Estes padrões de distribuição são esperados pelas condições fitogeográficas presentes no local de estudo, visto que áreas de floresta tropical de terras baixas a submontana (600 até $1500 \mathrm{~m}$ de altitude) geralmente apresentam mais espécies com ampla distribuição global (Gradstein \& Pócs 1989, Santos \& Costa 2010, Santos et al. 2011). Devido a adaptações como tolerância à dessecação e aos raios UV, espécies de briófitas com distribuição mais ampla tendem também a se dispersar efetivamente a longas distâncias, diferente das espécies endêmicas cujos diásporos são mais susceptíveis às mesmas condições (Van Zanten \& Pócs 1981, Santos \& Costa 2010).

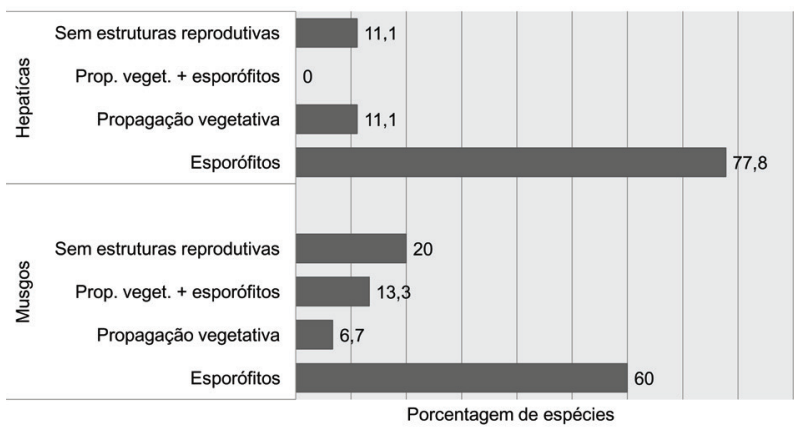

Fig. 5. Modos reprodutivos presentes nas briófitas da Estação Ecológica da UFMG (Belo Horizonte, Minas Gerais).

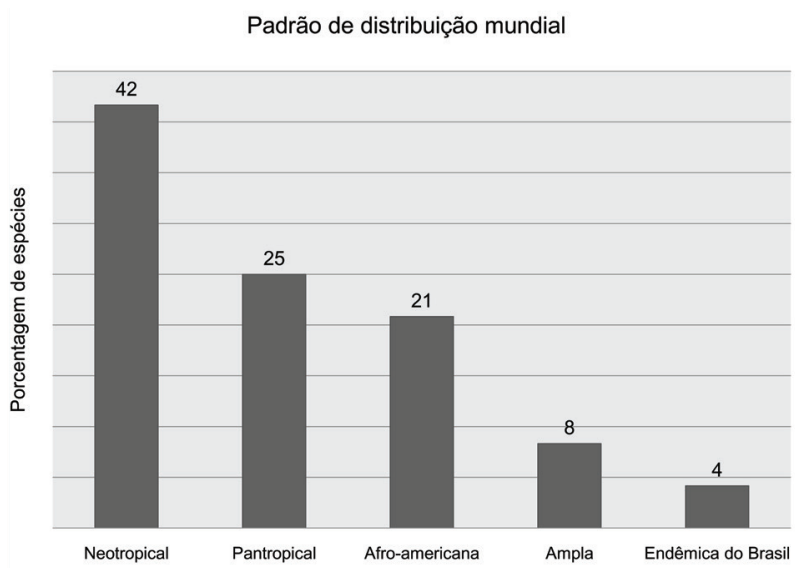

Fig. 6. Padrão de distribuição mundial das espécies encontradas na Estação Ecológica da UFMG (Belo Horizonte, Minas Gerais). 
Espaços de conservação são importantes para a manutenção da diversidade vegetal. A Estação Ecológica da UFMG cumpre este papel por abrigar múltiplas espécies da fauna e flora brasileira. Com este trabalho podemos ver tal importância em nível de brioflora, com pelo menos 24 espécies diferentes, sendo Isopterygium byssobolax um novo registro e a coleta após 160 anos da hepática Cylindrocolea planifolia para o estado de Minas Gerais. Além disso, visto que múltiplas formas de propagação das briófitas garantem o sucesso dessas plantas, ao registrar pela primeira vez propágulos assexuados no musgo Donnellia lagenifera amplia-se a compreensão sobre os mecanismos de reprodução e dispersão no grupo.

\section{AGRADECIMENTOS}

À Estação Ecológica da Universidade Federal de Minas Gerais e aos alunos que cursaram as disciplinas Metodologia de Campo (ICB015-2014.1) e Biologia de Briófitas (ICB054-2014.2) pelo auxílio durante as coletas. Ao Dr. John Spence e Dr. William Buck pelo auxílio com identificação taxonômica.

\section{REFERÊNCIAS}

Allen, B.H. 1992. A Revision of Ochrobryum (Leucobryaceae). Contributions University of Michigan Herbarium 18: 116-119.

Allen, B.H. 2002. Moss flora of Central America, Part 2. EncalyptaceaeOrthotrichaceae. Monogr. Syst. Bot. Missouri Bot. Gard. 90: 1-699.

Alvarenga, L.D.P. \& Lisboa, R.C.L. 2009. Contribuição para o conhecimento da taxonomia, ecologia e fitogeografia de Briófitas da Amazônia Oriental. Acta Amazonica 39(3): 495-504.

Alvarenga, L.D.P., Pôrto, K.C. \& Silva, M.P.P. 2009. Relations between regional-local habitat loss and metapopulation properties of epiphyllous bryophytes in the Brazilian. Atlantic Forest Biotropica 41(6): 682-691.

Álvares, É.S.S., Loyola, R.D. \& De Maria, M. 2005. A new species of the spider genus Aculepeira Chamberlin \& Ivie (Araneae: Araneidae) found in nests of Trypoxylon (Trypargilum) lactitarse Saussure (Hymenoptera: Sphecidae) from Minas Gerais, Brazil. Lundiana 6: $55-57$.

Álvares, É.S.S., Machado, E.O., Azevedo, C.S. \& De Maria, M. 2004. Composition of the spider assemblage in an urban forest reserve in southeastern Brazil and evaluation of, a two sampling method protocols of species richness estimates. Revista Ibérica de Aracnología 10: $185-194$

Antonini, Y. \& Martins, R.P. 2003. The Flowering-visiting bees at the ecological station of the Universidade Federal de Minas Gerais, Belo Horizonte, MG, Brazil. Neotropical Entomology 32(4): 565-575.

Aquino, H.F.D., Resende, I.L.D.M., Peralta, D.F. \& Rocha, L.M.D. 2015. Bryoflora of Gallery Forest in Quirinópolis, Goiás State, Brazil. Hoehnea 42(3): 419-424.

Barbosa-Campos, M.T. 2011. Morfoanatomia e ecofisiologia de Ouratea castaneifolia Engl. ocorrendo em diferentes ambientes lumínicos. Tese 51 f., Universidade Federal de Minas Gerais, Minas Gerais.

Bastos, C.J.P. 2015. Cheilolejeunea In Lista de Espécies da Flora do Brasil. Jardim Botânico do Rio de Janeiro. Disponível em: http:// floradobrasil.jbrj.gov.br/jabot/floradobrasil/FB97421. Acessado em 04.02. 2015.

Bastos, C.J.P. \& Yano, O. 2009. O gênero Lejeunea Libert (Lejeuneaceae) no estado da Bahia, Brasil. Hoehnea 36(2): 303-320.

Belotti, F.M. 2011. Capacidade de retenção de metais pesados pelo solo em área de implantação de estruturas de infiltração para águas pluviais urbanas em Belo Horizonte/MG. Tese 142 f., Universidade Federal de Minas Gerais, Minas Gerais.
Brito, E.S. \& Ilkiu-Borges, A.L. 2014. Briófitas de uma área de Terra Firme no município de Mirinzal e novas ocorrências para o estado do Maranhão, Brasil. Iheringia. Série Botânica 69: 133-142.

Buck, W.R. 1990. Contributions to the moss flora of Guyana. Memoirs of The New York Botanical Garden 64: 184-196.

1994. A sybiosis of the American species of Donnellia (Sematophyllaceae). Hikobia 11: 377-385.

1998. Pleurocarpous Mosses of the West Indies. Memoirs of the New York Botanical Garden 82: 1-400.

Câmara, P.E.A.S. 2015. Sematophyllaceae In Lista de Espécies da Flora do Brasil. Jardim Botânico do Rio de Janeiro. Disponível em: http:// floradobrasil.jbrj.gov.br/jabot/floradobrasil/FB96933. Acessado em 04.02.2015.

Campos, P.T. 2011. Fenologia e variações sazonais no status hídrico e na termotolerância em espécies lenhosas em um fragmento de mata semidecídua em Belo Horizonte, MG. Dissertação 89 f., Universidade Federal de Minas Gerais, Minas Gerais.

Carmo, D. M. D., \& Peralta, D. F. (2016). Survey of bryophytes in Serra da Canastra National Park, Minas Gerais, Brazil. Acta Botanica Brasilica, 30(2): 254-265.Costa, D.P. 1999. Epiphytic bryophyte diversity in primary and secondary lowland rainforests in southeastern Brazil. The Bryologist 102(2): 320-326.

2015a. Aneuraceae In Lista de Espécies da Flora do Brasil. Jardim Botânico do Rio de Janeiro. Disponível em: http://floradobrasil. jbrj.gov.br/jabot/floradobrasil/FB97824. Acessado em 04.02.2015.

2015b. Cephaloziellaceae In Lista de Espécies da Flora do Brasil. Jardim Botânico do Rio de Janeiro. Disponível em: http:// floradobrasil.jbrj.gov.br/jabot/floradobrasil/FB97231. Acessado em 04.02.2015.

2015c. Fossombroniaceae In Lista de Espécies da Flora do Brasil. Jardim Botânico do Rio de Janeiro. Disponível em: http:// floradobrasil.jbrj.gov.br/jabot/floradobrasil/FB97840. Acessado em 04.02.2015.

2015d. Pottiaceae In Lista de Espécies da Flora do Brasil. Jardim Botânico do Rio de Janeiro. Disponível em: http://floradobrasil. jbrj.gov.br/jabot/floradobrasil/FB96764. Acessado em 04.02.2015.

Costa, D.P., Bastos, C.J.P. \& Schäfer-Verwimp, A. 2015. Lejeuneaceae In Lista de Espécies da Flora do Brasil. Jardim Botânico do Rio de Janeiro. Disponível em: http://floradobrasil.jbrj.gov.br/jabot/ floradobrasil/FB97355. Acessado em 04.02.2015.

Costa, D.P. \& Santos, N.D. 2009. Conservação de Hepáticas na Mata Atlântica do sudeste do Brasil: uma análise regional no Estado do Rio de Janeiro. Acta Botânica Brasilica 23(4): 913-922

Costa, D.P., Santos, N.D. \& Váňa, J. 2008. A new species of Cylindrocolea (Cephaloziellaceae) from Brazil. The Bryologist 111(4): 667-669.

Crandall-Stotler, B. Stotler, R.E. \& Long, D.G. 2009. Morphology and classification of the Marchantiophyta In Bryophyte Biology. (B. Goffinet \& A.J. Shaw, eds.). Cambridge University Press, New York, p. 1- 54.

Costa, D. P., Peralta, D. F., Buck, W. R., Larrain, J., \& Von Konrat, M. A. T. T. 2017. Serra Do Curicuriari, Amazonas State, Brazil: The First Bryofloristic Analysis For A Brazilian Mountain In The Amazonian Forest. Phytotaxa, 303(3), 201-217.

Dal Pont, K.R. 2008. De "bota fora” à estação ecológica da UFMG (pequenas conquistas e a construção de pequenos significados ambientais urbanos). Dissertação, Universidade Federal de Minas Gerais, Minas Gerais.

Dias, P.G. \& Kumagai, A.F. 2011. Abundância e sazonalidade de Bittacidae (Mecoptera) em duas áreas de conservação em Minas Gerais, Brasil. Acta Biológica Paranaense 40(1-2): 33-37.

Florschütz-de Waard, J. \& Veling, K. 1996. Hypnaceae. In Flora of the Guianas, Series C: Bryophytes, Musci III. (A.R.A. Görts-Van Rijn, ed.). Royal Botanic Gardens, Richmond, Surrey, p. 439-462.

Frahm, J.P. 2003. Manual of tropical bryology. Tropical Bryology 23: $1-194$.

Fulford, M.H. 1976. Manual of the leafy Hepaticae of Latin América. Memoirs of the New York Botanical Garden 11: 393-535.

Florschütz-de Waard, K Veling and HR Zielman. 2011. Flora of the Guianas, Series C: 2 Bryophytes: Musci IV. Marion, J. Jansen-Jacobs (Eds). Royal Botanic Gardens. p 350-369. 
Germano, S.R. 2003. Florística e Ecologia das Comunidades de Briófitas em um Remanescente de Floresta Atlântica (Reserva Ecológica de Gurjaú, Pernambuco, Brasil). Tese 120 f., Universidade Federal de Pernambuco, Recife.

Glime, J.M. 2013. Sexual Strategies. In Bryophyte Ecology. Volume 1: Physiological Ecology. Ebook sponsored by Michigan Technological University and the International Association of Bryologists. Disponivel em: www.bryoecol.mtu.edu. Acessado em 27.08.2015.

Goffinet, B., Buck, W.R. \& Shaw, A.J. 2009. Morphology, anatomy, and classification of the Bryophyta. In Bryophyte Biology (B. Goffinet, \& A.J. Shaw, eds.). Cambridge University Press, New York, p. 55-138.

Gradstein, S.R. 1992. - Threatened bryophytes of the neotropical rain forest: a status report. Tropical Bryology p. 85-86.

1997. The taxonomic diversity of epiphyllous bryophytes. Abstracta Botanica 21(1): 15-19. 2011. A synopsis of the Frullaniaceae (Marchantiophyta) from Colombia. Caldasia, 33(2), 367-396.

Gradstein, S.R. \& Costa, D.P. 2003. The Hepaticae and Anthocerotae of Brazil. Memoirs of the New York Botanical Garden. New York Botanical Gradens Press, New York. 87: 1-318.

Gradstein, S.R., Churchill, S.P. \& Salazar, A. 2001. Guide to the bryophytes of tropical America. Memoirs of the New York Botanical Garden 86: $1-577$.

Gradstein, S.R. \& Pócs, T. 1989. Bryophytes. In Tropical rain forest ecosystems (H. Lieth \& M.J.A. Werger, eds.). Elsevier, Amsterdam, p. 311-325.

Henriques, D.K. \& Costa, D.P. 2015. Entodontaceae. Lista de Espécies da Flora do Brasil. Jardim Botânico do Rio de Janeiro. Disponível em: http://floradobrasil.jbrj.gov.br/jabot/floradobrasil/FB96243. Acessado em 04.02.2015.

Hijmans, R.J., Guarino, L., Bussink, C., Mathur, P., Cruz, M., Barrentes, I. \& Rojas, E. 2004. DIVA-GIS. Vsn. 5.0.A geographic information system for the analysis of species distribution data. Disponivel em: http://www.diva-gis.org/ Acessado em: 17.04.2017

Holz, I., \& Gradstein, R. S. 2005.Cryptogamic epiphytes in primary and recovering upper montane oak forests of Costa Rica-species richness, community composition and ecology. Plant Ecology 178(1): 89-109.

Holz, I., Gradstein, S.R., Heinrichs, J. \& Kappelle, M. 2002. Bryophyte diversity, microhabitat differentiation, and distribution of life forms in Costa Rican upper montane Quercus forest.The Bryologist 105(3): 334-348.

IEF-Instituto Estadual de Florestas. 2015. Cobertura vegetal de Minas Gerais. Disponível em: http://www.ief.mg.gov.br/florestas. Acessado em 08.07.2015

Ilkiu-Borges, A.L., Santos, R.C.P., Macedo, L.P.C., \& Pereira, M.A.V. 2009. As briófitas In Diversidade biológica das áreas de proteção ambiental: Ilhas do Combu e Algodoal-Maiandeua, Pará, Brasil (M.A.G. Jardim, org.). Três C. Gráfica e Editora, Belém, p. 227-244.

Ireland, R.R. \& Buck, W.R. 2009. Some Latin American Genera of Hypnaceae (Musci). Smithsonian Contributionsto Botany 93:1-97..

Julião, G.R., Fernandes, G.W., Negreiros, D., Bedê, L. \& Araújo, R.C. 2005. Insetos galhadores associados a duas espécies de plantas invasoras de áreas urbanas e peri-urbanas. Revista Brasileira de Entomologia 49(1): 97-106.

Kumagai, A.F. 2002. Os Ichneumonidae (Hymenoptera) da estação ecológica da Universidade Federal de Minas Gerais, Belo Horizonte, com ênfase nas espécies de Pimplinae. Revista Brasileira de Entomologia 46(2): 189-194.

Kürschner, H. 2004. Life strategies and adaptations in bryophytes from the Near and Middle East. Turkish Journal of Botany 28: 73-84

Longton, R.E. 1992. Reproduction and rarity in British mosses. Biological Conservation 59(2): 89-98.

Loyola, R.D. \& Martins, R.P. 2008. Habitat structure components are effective predictors of trap-nesting Hymenoptera diversity. Basic and Applied Ecology 9(6): 735-742.

Machado, R.B., Ramos Neto, M.B., Pereira, P.G.P., Caldas, E.F., Gonçalves, D.A., Santos, N.S., Tabor, K. \& Steininger, M. 2004. Estimativas de perda da área do Cerrado brasileiro. Brasília. Conservação Internacional. Brasília, Distrito Federal. 23 p.

Maciel-Silva, A. S., Valio, I. F. M., \& Rydin, H. 2012. Altitude affects the reproductive performance in monoicous and dioicous bryophytes: examples from a Brazilian Atlantic rainforest. AoB plants v2012: pls 016. DOI: https://doi.org/10.1093/aobpla/pls016

Moura, O.S.D., Ilkiu-Borges, A.L. \& Brito, E.D.S. 2013. Bryoflora (Bryophyta and Marchantiophyta) of Combu Island, Belém, Pará State, Brazil. Hoehnea 40(1): 143-165.

Oki, Y., Soares, N. Belmiro, M.S., Corrêa Junior, A. \& Fernandes, G.W. 2009. Influência dos fungos endofíticos sobre os herbívoros de Baccharis dracunculifolia (Asteraceae). Neotropical Biology and Conservation 4(2): 83-88.

Oliveira, M.P, Carmo, E.C.M. \& Maciel-Silva, A.S. 2014. Moss selfie with a digital microscope - Taking photos of a moss with an USB digital microscope: a good alternative strategy to obtain high magnification images. Microbe Hunter Microscopy Magazine 44: 6-11.

Oliveira, S.M. \& Pôrto, K.C. 1998. Reprodução sexuada em musgos acrocárpicos do Estado de Pernambuco, Brasil. Acta Botanica Brasílica 12(3): 385-392.

Peralta, D.F. 2015a. Bryaceae In Lista de Espécies da Flora do Brasil. Jardim Botânico do Rio de Janeiro. Disponível em: http://floradobrasil. jbrj.gov.br/jabot/floradobrasil/FB95964. Acessado em 04.02.2015. . 2015b. Calymperaceae In Lista de Espécies da Flora do Brasil. Jardim Botânico do Rio de Janeiro. Disponível em http://floradobrasil. jbrj.gov.br/jabot/floradobrasil/FB96030. Acessado em 04.02.2015.

.2015c. Erpodiaceae In Lista de Espécies da Flora do Brasil. Jardim Botânico do Rio de Janeiro. Disponível em: http://floradobrasil. jbrj.gov.br/jabot/floradobrasil/FB96274. Acessado em 04.02.2015.

. 2015d. Frullaniaceae In Lista de Espécies da Flora do Brasil. Jardim Botânico do Rio de Janeiro. Disponível em: http://floradobrasil. jbrj.gov.br/jabot/floradobrasil/FB97292. Acessado em 04.02.2015.

. 2015e. Hypnaceae In Lista de Espécies da Flora do Brasil. Jardim Botânico do Rio de Janeiro. Disponível em: http://floradobrasil. jbrj.gov.br/jabot/floradobrasil/FB96434. Acessado em 04.02.2015.

2015f. Leucobryaceae In Lista de Espécies da Flora do Brasil. Jardim Botânico do Rio de Janeiro. Disponível em: http://floradobrasil. jbrj.gov.br/jabot/floradobrasil/FB96496. Acessado em 04.02.2015.

.2015g. Stereophyllaceae In Lista de Espécies da Flora do Brasil. Jardim Botânico do Rio de Janeiro. Disponível em: http://floradobrasil. jbrj.gov.br/jabot/floradobrasil/FB97114. Acessado em 04.02.2015.

Proctor, M.C.P. 2000. Physiological ecology. In Bryophyte Biology (A.J. Shaw, \& B. Goffinet, eds.). Cambridge University Press, Cambridge, p. $225-247$

Reiner-Drehwald, M.E. \& Goda, A. 2000. The Journal of the Hattori Botanical Laboratory 89:1-54.

Reiner-Drehwald, M.E. 2000. Las Lejeuneaceae (Hepaticae) de Misiones, Argentina. VI. Lejeunea y Taxilejeunea. Tropical Bryology 19: $81-131$.

2003. Lejeunea. In The Hepaticae and Anthocerotae of Brazil (S.R. Gradstein, \& D.P. Costa, eds.). Memoirs of the New York Botanical Garden. New York Botanical Gradens Press, New York. p. 155-163

Santos, N.D.D. \& Costa, D.P.D. 2010. Phytogeography of the liverwort flora of the Atlantic Forest of southeastern Brazil. Journal of Bryology 32(1): 9-22.

Santos, N.D.D., Costa, D.P.D., Kinoshita, L.S. \& Shepherd, G.J. 2011. Aspectos brioflorísticos e fitogeográficos de duas formações costeiras de Floresta Atlântica da Serra do Mar, Ubatuba/SP, Brasil. Biota Neotropica 11(2): 425-438

Schofield, W.B. 1985. Introduction to bryology. Macmillan, New York. p. 423

Sousa, R.V.D., \& Câmara, P.E.A.S. 2015. Survey of the bryophytes of a gallery forest in the National Park of Serra do Cipó, Minas Gerais, Brazil. Acta Botanica Brasilica 29(1): 24-29.

Souza-Silva, M., Fontenelle, J.C.R. \& Martins, R.P. 2001. Composição, abundância e sazonalidade de dípteros visitantes florais. Neotropical Entomology 30(3): 351-359.

Tavares, V.C., Aguiar, L.M.S., Perini, F.A., Falcão, F.C. \& Gregorin, R. 2010. Bats of the state of Minas Gerais, southeastern Brasil. Chiroptera Neotropical 16(1): 675-705.

Tonini, A., Starling, M.F.V., Yano, O. \& Figueiredo, J.A. 2005. Briofitas das Ilhas do Passatempo, Parque Municipal das Mangabeiras, Belo Horizonte, MG. Bios, Cadernos do Departamento de Ciências Biológicas da Pontifícia Universidade Católica de Minas Gerais 13: 25-34. 
Universidade Federal de Minas Gerais - UFMG 2015. Estação Ecológica da UFMG. Disponível em: www.ufmg.br/estacaoecologica/. Acessado em 01.05.2015.

Van Zanten, B.O. \& Pócs T. 1981. Distribution and dispersal of bryophytes. Advances in Bryology 1: 479-562.

Yano, O., Peralta, D.F. \& Bordin, J. 2010. Musgos dos estados de Alagoas, Bahia, Pernambuco e Sergipe, Brasil, depositados no herbário SP. Hoehnea 37: 211-265.
2011. Antóceros e hepáticas dos estados de Alagoas, Bahia, Pernambuco e Sergipe, Brasil, depositados no herbário SP. Hoehnea 38: 429-481.

Yano, O. 2010. Levantamento de novas ocorrências de briófitas brasileiras. Instituto de Botânica. Disponível em: http://www. ibot. sp. gov. br/ publicacoes/virtuais/briofitas. pdf $>$. Acessado em 01.05.2015. 